\title{
Prevalência do Transtorno do Desenvolvimento da Coordenaçáo em uma amostra de crianças brasileiras $^{1}$
}

\author{
Thais Silva Beltrame, Renata Capistrano, Juliano Maestri Alexandre, Tailine Lisboa, \\ Rubian Diego Andrade, Érico Pereira Gomes Felden
}

Universidade do Estado de Santa Catarina - UDESC, Florianópolis, SC, Brasil.

\begin{abstract}
Resumo: Introdução: Devido às consequências que o Transtorno do Desenvolvimento da Coordenação - TDC pode trazer para o desenvolvimento da criança nos aspectos motores, afetivos e sociais, há necessidade de investigações com amostras representativas no Brasil. Objetivo: Este estudo objetivou investigar a prevalência do TDC em escolares de 7 a 10 anos de idade. A amostra foi composta por 787 crianças do município de Florianópolis/SC. Método: O desempenho motor foi avaliado por meio da Movement Assessment Battery for Children Second Edition - MABC-2 que classifica o desempenho motor em três: dificuldade, risco e desempenho motor normal. Para as análises, recorreu-se à estatística descritiva (médias, mínimos, máximos, desvio padrão, frequências) e inferencial (teste Quiquadrado, U de Mann-Whitney, Krusskal-Wallis com post-hoc de Dunn). A significância estatística adotada foi de $\mathrm{p}<0,05$. Resultados: A prevalência de dificuldade motora e risco para dificuldade motora foi de 7,1\% e $11,3 \%$, respectivamente, e sem dificuldade de movimento foi $81,6 \%$. Ao associar o sexo com as classificações motoras, observou-se que os meninos apresentaram mais dificuldade motora $\left(X^{2}=6,38 ; p=0,04\right)$. As meninas apresentaram mais dificuldade nas habilidades de lançar e receber e os meninos nas habilidades de destreza manual. As crianças na faixa etária de 7 e 8 anos apresentaram prevalências maiores de TDC. Conclusão: A prevalência do transtorno, bem como as diferenças entre os sexos, foi considerada semelhante às evidências apresentadas em pesquisas internacionais.
\end{abstract}

Palavras-chave: Habilidade Motora, Transtorno das Habilidades Motoras, Crianças.

\section{Prevalence of Developmental Coordination Disorder in a sample of Brazilian children}

\begin{abstract}
Introduction: Due the consequences that the Developmental Coordination Disorder - DCD can bring to the development of children in motor, affective and social aspects, a research with representative samples in Brazil becomes needed. Objective: This study aimed to investigate the prevalence of DCD in students aged 7 to 10 years old. The sample consisted of 787 children from the city of Florianópolis/SC. Method: The motor performance was evaluated by the Movement Assessment Battery for Children Second Edition - MABC-2 which ranks the motor performance in three: difficulty, risk and standard motor performance. For analysis we used descriptive statistics (average, minimum, maximum, standard deviation, and frequency) and inferential statistics (Chi-square test, Mann-Whitney, Krusskal-Wallis with post-hoc Dunn). The statistical significance was set on $\mathrm{p}<0.05$. Results: The prevalence of motor difficulty and risk for motor difficulty was $7.1 \%$ and $11.3 \%$ respectively and with no motor difficulty was $81.6 \%$. By associating sex with motor classifications it was observed that boys had more motor difficulty $(\mathrm{X} 2=6.38$; $\mathrm{p}=0.04)$. Girls showed more difficulty with the throwing and receiving skills; and the boys with manual dexterity skills. Children aged 7 and 8 years had higher prevalence of DCD. Conclusion: The prevalence of the disorder, as well as the differences between the sexes was considered similar to evidences presented in international research.
\end{abstract}

Keywords: Motor Skills, Motor Skills Disorders, Children.

Autor para correspondência: Renata Capistrano, Centro de Ciências da Saúde e do Esporte, Rua Pascoal Simone, 358, Coqueiros, CEP 88080-350, Florianópolis, SC, Brasil, e-mail: recapis@gmail.com

Recebido em Dez. 22, 2015; $1^{\text {a }}$ Revisão em Maio 9, 2016; $2^{\text {a }}$ Revisão em Jul. 7, 2016; Aceito em Ago. 4, 2016. 


\section{Introdução}

O movimento para crianças que apresentam dificuldades motoras pode se constituir em um grande desafio, de maneira que determinadas tarefas típicas da infância se tornam bastante difíceis (CAÇOLA et al., 2015; FARHAT et al., 2015). Entre as dificuldades motoras, encontra-se o Transtorno do Desenvolvimento da Coordenação - TDC.

O TDC pode ser identificado quando, na ausência de distúrbios físicos/neurológicos conhecidos, há um significativo prejuízo no desenvolvimento das habilidades motoras, atividades acadêmicas, de lazer e de vida diária, considerando a idade cronológica e nível cognitivo da criança (AMERICAN..., 2013). Para a identificação desse distúrbio motor, são necessários testes motores válidos e confiáveis (NIEMEIJER; WAELVEDE; SMITS-ENGELSMAN, 2015).

O MABC-2 é um dos instrumentos mais utilizados para indicar dificuldade significativa de movimento (CAPISTRANO et al., 2015). Crianças com essa característica apresentam atrasos no desenvolvimento das habilidades motoras, prejudicando a realização de atividades escolares e do cotidiano, parecendo desajeitadas ou mesmo atrapalhadas motoramente (CAÇOLA et al., 2015; FARHAT et al., 2015). Esses prejuízos muitas vezes podem estar relacionados com a percepção de baixa autoeficácia, dificuldades de relacionamento e ao baixo interesse em atividades físicas (VALENTINI et al., 2012).

$\mathrm{Na}$ faixa etária dos 7 aos 10 anos, o movimento se torna uma ferramenta aplicada a uma série de atividades de movimentos complexos para a vida diária, recreação e resultados esportivos. Esse é um período em que as habilidades estabilizadoras, locomotoras e manipulativas fundamentais são progressivamente refinadas, combinadas e elaboradas para o uso em situações de crescente demanda (GALLAHUE; OZMUN; GOODWAY, 2013). Entretanto, para crianças que apresentam TDC, determinadas tarefas típicas da infância, como atividades de autocuidado, tarefas motoras e escolares, tornam-se um grande desafio (RIVILIS et al., 2011; AMERICAN..., 2013; BIEBARA et al., 2016).

As estimativas internacionais apontam para uma prevalência do transtorno em torno de $6 \%$ das crianças em idade escolar, com destaque para o sexo masculino (ORGANIZAÇÃO..., 1993; AMERICAN..., 2013). Estudos no Reino Unido, Alemanha, Holanda, Canadá evidenciaram prevalências de 5,2\% a 7,7\% de dificuldades motoras entre crianças (BLANK et al., 2012; LINGAM et al., 2010; CAIRNEY et al.,
2005; JONGMANS; SMITS-ENGELSMAN; SHOEMAKER, 2003).

Nos estudos brasileiros, não há um consenso da estimativa do Transtorno do Desenvolvimento da Coordenação, com as prevalências variando em torno de $4,4 \%$ a $19,9 \%$ (SANTOS; VIEIRA, 2013; SOUZA et al., 2007; VALENTINI et al., 2012). Esta dissonância justifica a necessidade de maior aprofundamento em investigaçóes na área do comportamento motor.

Devido às consequências que o TDC pode trazer para o desenvolvimento da criança nos aspectos motores, afetivos e sociais, há necessidade de investigaçóes com amostras representativas no Brasil. Diante disso, este estudo objetivou investigar a prevalência de transtorno do desenvolvimento da coordenaçáo em escolares de 7 a 10 anos de idade.

\section{Método}

Trata-se de uma análise secundária de dados realizada a partir de pesquisas desenvolvidas no Laboratório de Distúrbio da Aprendizagem e do Desenvolvimento - LADADE da Universidade do Estado de Santa Catarina - Brasil, realizada no município de Florianópolis/SC.

\subsection{Participantes do estudo}

Participaram deste estudo crianças da cidade de Florianópolis/SC, capital do estado, com uma população de 421.240 habitantes, que apresenta elevado Índice de Desenvolvimento Humano geral (IDHM 0,847) e também nas três dimensões que compóem esta estimativa: área de educação $(0,800)$, renda $(0,870)$ e longevidade $(0,873)$ (ORGANIZAÇÃO..., 2010). A população estimada deste estudo é em torno de 7000 crianças (INSTITUTO..., 2012). Para o cálculo amostral, considerou-se um erro amostral de $4 \%$, nível de confiança de $95 \%$, prevalência desconhecida de $50 \%$ e acrescentaram-se $10 \%$ para possíveis perdas e recusas. Com a equação de Luiz e Magnanini (2000), estimou-se a participação de 599 crianças. No entanto, participaram 787 escolares de seis escolas públicas de ambos os sexos, sendo $48,8 \%$ do sexo masculino com média de idade de $8,67(1,1)$ anos.

Os critérios de inclusão considerados foram: intenção dos escolares para participação voluntária; faixa etária estabelecida (7 a 10 anos e 11 meses e 29 dias); apresentação da autorização dos pais ou responsáveis por meio do Termo de Consentimento Livre e Esclarecido; ausência de deficiências físicas 
e/ou mentais (indicados pelos professores regentes e de Educação Física).

Foram excluídas dessa amostra crianças que estivessem em intervençôes terapêuticas.

\subsection{Instrumentos}

Para avaliação motora, utilizou-se a Movement Assessement Battery for Children - MABC-2. (HENDERSON; SUGDEN; BARNETT, 2007). Este instrumento é composto pelo checklist que avalia o movimento em contextos diferentes e os testes motores. É a bateria motora mais utilizada para identificar risco e dificuldades motoras (CAPISTRANO et al., 2015), a qual já foi validada para a população brasileira (VALENTINI; RAMALHO; OLIVEIRA, 2014). Destaca-se que o instrumento deve ser usado como uma ferramenta de rastreio preditivo para distúrbios motores (HENDERSON; SUGDEN; BARNETT, 2007).

Esta avaliaçáo motora tem sido utilizada em diversas nacionalidades para identificar crianças com dificuldade significativa de movimento, como na China (CHANG; YU, 2016), Japão (KITA et al., 2016), Holanda (NIEMEIJER et al., 2015) e Brasil (VALENTINI et al., 2014). Trata-se de um teste motor com três conjuntos de tarefas (destreza manual, lançar e receber e equilíbrio), para as faixas etárias específicas: faixa um ( 3 a 6 anos); faixa dois (7 a 10 anos) e faixa três (11 a 16 anos). Para cada faixa etária, são estabelecidas tarefas diferenciadas, de acordo com os níveis diferentes de complexidade. Neste estudo, especificamente, foi utilizada a faixa etária dois (7 a 10 anos) (Tabela 1). As crianças com pontuaçáo total abaixo do $5^{\circ}$ percentil foram consideradas com indicativo de dificuldade de movimento; valores entre o $5^{\circ}$ e o $15^{\circ}$ percentil indicam que a criança tem risco de dificuldade motora; e valores acima do $15^{\circ}$ percentil indicam um desenvolvimento motor normal.

\subsection{Procedimento de coleta de dados}

Para a coleta de dados de cada banco foi obtida a aprovação do Comitê de Ética e Pesquisa em Seres Humanos da Universidade do Estado de Santa Catarina (CEPSH/UDESC) de acordo com a resolução no 466/12, sob os respectivos números de parecer 16449913.8.0000.0118, 38772214.3.0000.0118 e 38/2009.

Depois da entrega do Termo de Consentimento Livre e Esclarecido - TCLE, devidamente assinado por responsável, as coletas foram iniciadas. As avaliaçôes motoras foram realizadas nas escolas, de forma individual e em ambientes que propiciaram o mínimo de interferências externas, levando aproximadamente 40 minutos por escolar. Todos os avaliadores receberam capacitaçóes padronizadas anteriormente às coletas no laboratório responsável pela pesquisa sob a coordenaçáo de dois doutores da área, a fim de uniformizar os procedimentos e minimizar as possíveis disparidades entre avaliadores.

O retorno das informaçóes à escola e aos alunos e seus responsáveis foi por meio de relatórios entregues em palestras agendadas de acordo com o calendário de cada escola, privilegiando datas que contemplavam entrega de boletins e reuniáo de pais. Nesses encontros, com o objetivo de não gerar constrangimento às crianças com TDC, focou-se uma abordagem geral sobre a saúde do escolar em um primeiro momento e, em seguida, foi dada ênfase aos transtornos motores e repercussóes para as atividades de vida diária. Participaram desses encontros pediatras, nutricionistas, psicólogos, educador físico e especial. Destaca-se que desde 2013, o LADADE oferta projetos de extensão para crianças com dificuldades motoras e estas são provenientes das pesquisas realizadas em ambiente escolar. Além disso, foram dadas orientaçóes para os professores com o objetivo de melhorar o dia a dia das crianças identificadas com TDC.

Tabela 1. Tarefas para avaliação de cada habilidade do teste motor para faixa etária de 7 a 10 anos. Movement Assessment Battery for Children - Second Edition (MABC-2).

\begin{tabular}{lll}
\hline \multicolumn{1}{c}{ Habilidades } & \multicolumn{1}{c}{ Tarefas } & \multicolumn{1}{c}{ Registros } \\
\hline \multirow{2}{*}{ Destreza Manual } & Colocando Pinos & Tempo em segundos \\
& Passando o Cordão & Tempo em segundos \\
& Trilha da Bicicleta & Número de erros \\
\hline \multirow{2}{*}{ Lançar e Receber } & Lançando e Recebendo com as duas mãos & Número de recebimentos corretos \\
& Lançando o saco de feijão & Número de acertos no alvo \\
\hline \multirow{2}{*}{ Equilíbrio Estático e } & Equilíbrio sobre a tábua & Tempo em segundos \\
Dinâmico & Andando sobre a linha & Número de passos corretos \\
& Saltanto em tapetes & Número de passos corretos \\
\hline
\end{tabular}




\subsection{Análise estatística}

O programa estatístico utilizado para análise foi o software Statistical Package for Social Sciences - SPSS, versão 20.0. A análise descritiva foi realizada por meio de médias, frequências e desvios padrão. Para verificação da normalidade da distribuição dos dados, utilizou-se o teste de Kolmogorov-Smirnov. Nas análises inferenciais, utilizou-se o teste de Quiquadrado para as associaçôes entre as variáveis categóricas. Para a diferença de médias entre variáveis contínuas, recorreu-se ao teste U de Mann-Whitney para dois grupos e Krusskal-Wallis com post-hoc de Dunn para três. Além disso, foi utilizado o teste de diferença entre proporçóes no software Medcalc, versão 9.1.0.1. Para todas as análises, adotou-se um nível de confiança de $95 \%(\mathrm{p}<0,05)$.

\section{Resultados}

A prevalência de crianças com risco e dificuldade de movimento foi de $11,3 \%$ e $7,1 \%$, respectivamente. Já o percentual daqueles sem dificuldade de movimento foi $81,6 \%$. Ao associar o sexo com as classificaçóes motoras (sem dificuldade de movimento, risco e dificuldade de movimento) observou-se que os meninos $(9,1 \%)$ apresentaram uma tendência a ter dificuldade motora
$\left(\mathrm{X}^{2}=6,38 ; \mathrm{p}=0,04\right)$ quando comparados às meninas. No entanto, quando estratificado por classificaçáo motora (sem dificuldade de movimento, risco e dificuldade de movimento) náo foram observadas diferenças significativas considerando as proporçóes entre os sexos (Figura 1).

$\mathrm{Na}$ Tabela 2, são apresentados os valores médios da pontuação total do MABC-2 de acordo com o sexo e idade. Não foram encontradas diferenças entre meninos e meninas em todas as faixas etárias ( $p>0,05)$. Quando analisados separadamente meninos em todas as idades, não foram identificadas diferenças entre as faixas etárias $(\mathrm{p}=0,100)$. O mesmo comportamento foi encontrado para as meninas $(\mathrm{p}=0,834)$.

A prevalência de risco de dificuldade de movimento nos meninos tem um aumento no intervalo de 7 a 8 anos de idade e uma queda a partir de 9 e 10 anos. O mesmo comportamento foi encontrado para o indicativo de dificuldade de movimento (Figura 2). Diferentemente dos meninos, o risco de dificuldade de movimento das meninas apresentou comportamento constante em todas as idades e valores semelhantes para o indicativo de dificuldade de movimento no intervalo de 7 e 8 anos e uma queda no período de 8 a 10 anos (Figura 3).

Quando observado o desempenho motor geral, verifica-se que aos 7 e 8 anos tanto meninos quanto

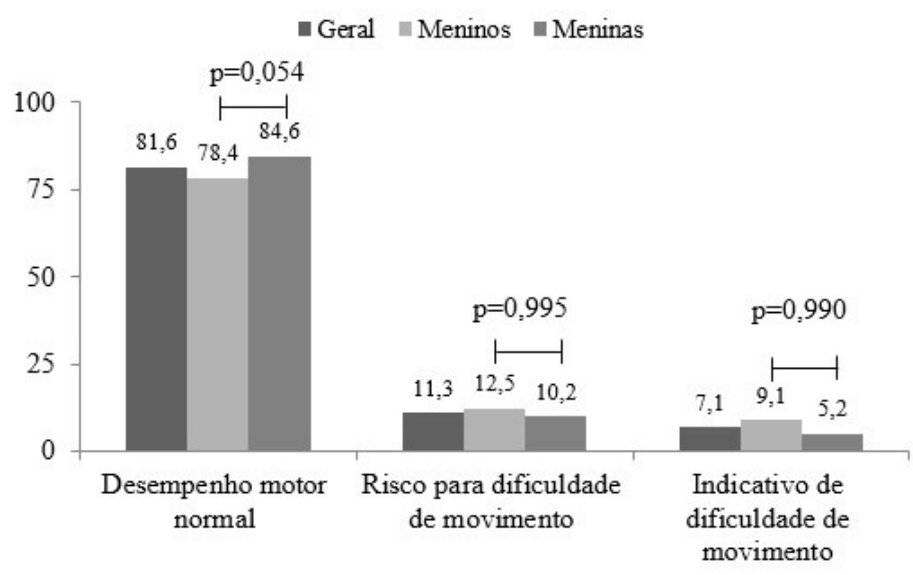

Figura 1. Classificação do desempenho motor geral e separado por sexo.

Tabela 2. Diferença da pontuação total do teste MABC-2 separado por idade e sexo.

\begin{tabular}{cccc}
\hline \multirow{2}{*}{ Idade } & \multicolumn{2}{c}{ Sexo } & \multirow{2}{*}{ p-valor* } \\
\cline { 2 - 3 } & Meninos M(DP) & Meninas M(DP) & 0,782 \\
7 anos & $72,6(13,4)$ & $72,6(10,6)$ & 0,098 \\
8 anos & $69,7(12,3)$ & $72,7(10,7)$ & 0,524 \\
9 anos & $72,6(11,7)$ & $73,9(8,9)$ & 0,831 \\
10 anos & $73,7(10,3)$ & $73,5(9,7)$ & - \\
p-valor** & 0,100 & 0,834 & \\
\hline
\end{tabular}

M - média; DP - desvio padrão; *teste U Mann-Whitney; ** teste Kruskal-Wallis. 
meninas apresentam mais dificuldade de movimento (Figura 4).

Quando analisadas a classificação motora do grupo de meninas e meninos por faixa etária, verificou-se que não foram encontradas associaçôes entre os sexos ( $p>0,05)$ (Tabela 3$)$.

Em relaçáo às tarefas de destreza manual, os meninos apresentaram diferenças significativas entre as idades de 7 a 10 anos $(\mathrm{p}=0,045)$. Quando feita a análise do post-hoc, foram identificadas diferenças apenas entre meninos com 7 e 8 anos $(\mathrm{p}=0,047)$, indicando um melhor desempenho aos sete anos. Verificou-se também que apresentaram diferenças nas tarefas de equilíbrio na faixa etária investigada $(\mathrm{p}=0,007)$, sobretudo entre as idades de 7 e $10(\mathrm{p}=0,019), 8$ e $10(\mathrm{p}=0,029)$, apresentando aumento no desempenho com o avançar da idade. Ainda considerando apenas os meninos, não foram

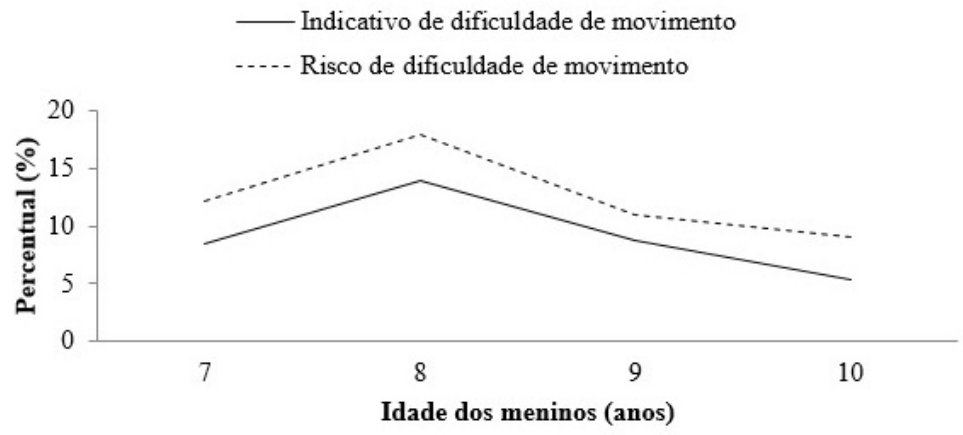

Figura 2. Classificação do desempenho motor dos meninos por idade.

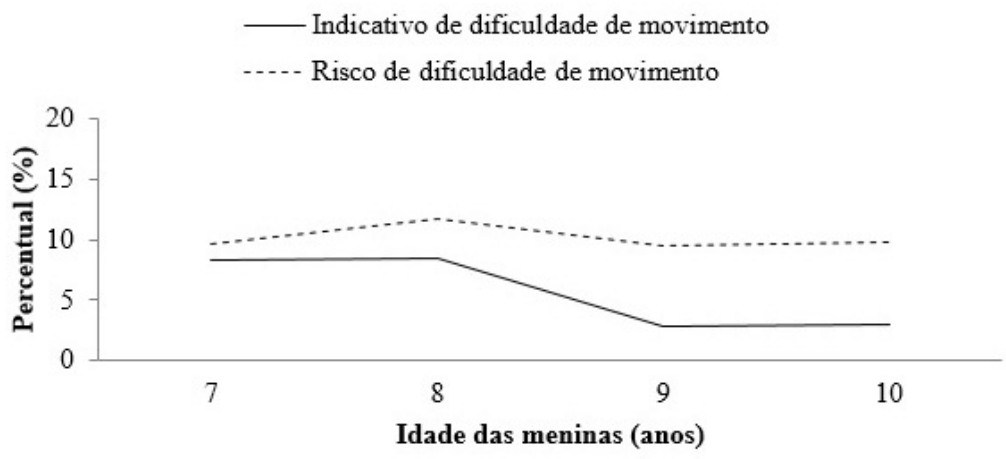

Figura 3. Classificação do desempenho motor das meninas por idade.

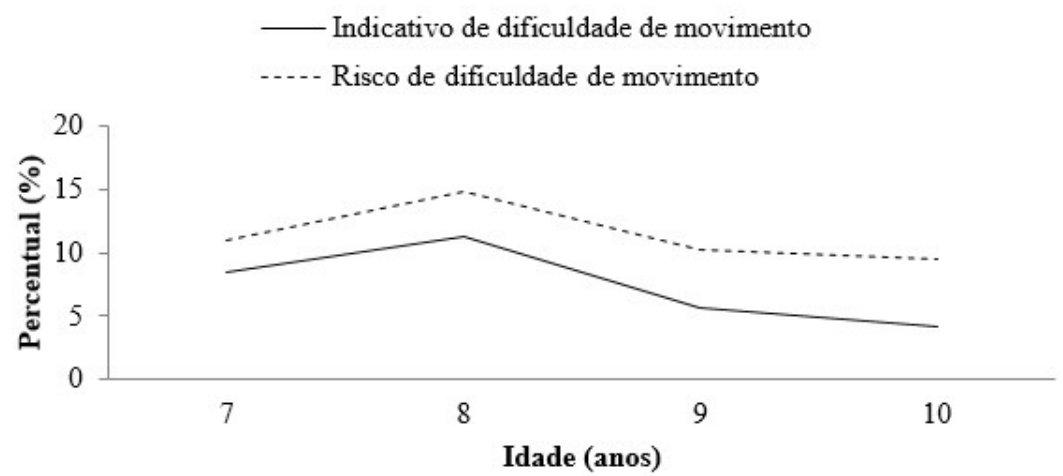

Figura 4. Classificação do desempenho motor geral separado por idade. 
encontradas diferenças entre as faixas etárias para as tarefas de lançar e receber $(\mathrm{p}=0,053)$ e pontuação total do teste $(\mathrm{p}=0,100)$. As meninas apresentaram diferenças significativas nas tarefas de equilíbrio nas diferentes faixas etárias $(\mathrm{p}=0,009)$. Com a análise do post-hoc, as diferenças foram identificadas nas idades de 7 e 10 anos ( $\mathrm{p}=0,007)$, demonstrando melhora das médias com o aumento da idade. Já nas outras tarefas e na pontuação total do teste não foram encontradas diferenças significativas entre as meninas nas faixas etárias analisadas (Tabela 4).

\section{Discussão}

Os estudos na interface entre comportamento motor e saúde coletiva buscam descrever as reais prevalências dos diferentes distúrbios motores na população. Especificamente com relação ao TDC, é possível identificar prevalências divergentes considerando estudos com amostras nacionais e internacionais, assim como considerando a abrangência dos estudos. Neste contexto, constatou-se na amostra de crianças de Florianópolis, uma prevalência de $7,1 \%$ de TDC. Tal prevalência vai ao encontro de estudos internacionais e apresenta certa divergência com estudos nacionais. Na Holanda, um estudo que avaliou 749 crianças, evidenciou 7,7\% delas com dificuldade motora (JONGMANS; SMITSENGELSMAN; SHOEMAKER, 2003). Já em pesquisa realizada no Canadá, com 564 crianças, apurou-se um percentual de $7,5 \%$ de dificuldade significativa de movimento (CAIRNEY et al., 2005).

Destaca-se, nesse ínterim, a dificuldade de avaliação do TDC em amostras populacionais, considerando que os critérios de avaliação mais aceitos pela literatura constituem-se de um conjunto de testes motores que

Tabela 3. Classificação motora dos meninos e meninas por idade.

\begin{tabular}{cccc}
\hline Idade & $\begin{array}{c}\text { Risco de dificuldade de } \\
\text { movimento (\%) }\end{array}$ & $\begin{array}{c}\text { Indicativo de dificuldade de } \\
\text { movimento (\%) }\end{array}$ & p-valor* \\
\hline 7 anos & & & 0,883 \\
Meninos & 12,2 & 8,5 & \\
Meninas & 9,7 & 8,3 & 0,175 \\
\hline 8 anos & & & \\
Meninos & 18,0 & 14,0 & 0,176 \\
Meninas & 11,7 & 8,5 & \\
\hline 9 anos & 11,0 & 8,8 & 0,642 \\
Meninos & 9,5 & 2,9 & \\
Meninas & & & 5,4 \\
\hline 10 anos & 9,0 & 3,0 & \\
Meninos & 9,8 &
\end{tabular}

*p-valor do teste Quiquadrado.

Tabela 4. Pontuação nos testes motores separados por idade e sexo.

\begin{tabular}{|c|c|c|c|c|}
\hline \multirow{2}{*}{ Idade } & \multicolumn{3}{|c|}{ Testes motoras } & \multirow{2}{*}{ Pontuação tota } \\
\hline & Destreza manual & Lançar e receber & Equilíbrio & \\
\hline \multicolumn{5}{|l|}{7 anos } \\
\hline Meninos & $23,5(6,6)$ & $18,36(5,1)$ & $30,3(6,8)$ & $72,7(13,4)$ \\
\hline Meninas & $24,5(5,8)$ & $16,6(4,7)$ & $31,4(6,1)$ & $73,6(10,5)$ \\
\hline p-valor & 0,539 & 0,027 & 0,038 & 0,782 \\
\hline \multicolumn{5}{|l|}{8 anos } \\
\hline Meninos & $21,1(6,8)$ & $18,2(5,1)$ & $30,4(6,6)$ & $69,6(12,3)$ \\
\hline Meninas & $23,2(6,3)$ & $16,8(5,1)$ & $32,6(4,8)$ & $72,7(10,7)$ \\
\hline p-valor & 0,036 & 0,048 & 0,026 & 0,098 \\
\hline \multicolumn{5}{|l|}{9 anos } \\
\hline Meninos & $21,6(6,6)$ & $19,7(4,2)$ & $31,4(4,9)$ & $72,6(11,7)$ \\
\hline Meninas & $23,9(6,1)$ & $17,6(3,4)$ & $32,5(4,7)$ & $74,0(8,9)$ \\
\hline p-valor & 0,013 & $<0,001$ & 0,044 & 0,524 \\
\hline \multicolumn{5}{|l|}{10 anos } \\
\hline Meninos & $21,7(5,9)$ & $19,7(4,8)$ & $32,6(5,3)$ & $73,8(10,3)$ \\
\hline Meninas & $22,9(5,9)$ & $16,9(4,3)$ & $33,8(4,0)$ & $73,5(9,6)$ \\
\hline p-valor & 0,123 & $<0,001$ & 0,173 & 0,831 \\
\hline
\end{tabular}

p-valor do teste U de Mann Whitney. 
necessitam, além de equipe qualificada, controle de variáveis contextuais e de tempo razoável de aplicação. Diante disso, acredita-se que a semelhança entre os dados deste estudo e dos resultados encontrados em pesquisas internacionais são explicados pelo tamanho da amostra, que possivelmente minimiza resultados com vieses de seleção locais.

Diferentemente do presente estudo, a maioria dos estudos brasileiros, quando comparados aos internacionais, apresenta índices de dificuldade motora superiores. Em Florianópolis, Silva e Beltrame (2013) avaliaram 406 crianças sendo que 11,1\% apresentaram dificuldade significativa de movimento. Em Maringá, 581 crianças foram avaliadas, sendo que $11,4 \%$ apresentam dificuldade motora (SANTOS; VIEIRA, 2013). Considerando que a prevalência do TDC é diferente por subgrupos, por exemplo, entre idade e sexo, amostras ampliadas tendem a diluir superestimaçóes da variável principal, sendo que quanto maior a amostra, maior a probabilidade de ela refletir a população inteira (FIELD, 2009).

$\mathrm{Na}$ amostra analisada do presente estudo, identificou-se prevalência de TDC superior nos meninos. Dados da Organização Mundial de Saúde (OMS) indicam que a dificuldade motora ocorre com maior frequência em meninos - proporção de quatro ou cinco meninos para cada menina. Confirmando essa tendência, Miranda, Beltrame e Cardoso (2011) avaliaram 380 escolares em Florianópolis/SC e os meninos apresentaram média inferior no desempenho motor quando comparado ao grupo de meninas. Green et al. (2011), ao avaliarem 4.331 crianças inglesas, identificaram 173 delas com indicativo de dificuldade motora, sendo $60,6 \%$ meninos e $40,4 \%$ meninas. As maiores dificuldades observadas nos meninos foram nas habilidades de destreza manual, ao contrário das meninas que apresentaram mais dificuldade nas habilidades de lançar e receber.

A literatura vem apresentando possíveis explicações para essas diferenças. Os meninos, culturalmente são incentivados a praticarem atividades mais vigorosas e que exijam coordenação ampla, não sendo tão desenvolvidas as habilidades finas, enquanto que as meninas optam por atividades mais calmas como brincar com bonecas, desenhar, entre outras (SILVA et al., 2014; FARIAS JÚNIOR et al., 2012; COUTINHO; SPESSATO; VALENTINI, 2011).

Quando analisado o comportamento do desempenho motor por idade, observou-se maior porcentagem de meninos e meninas na faixa etária de 7 a 8 anos com indicativo de dificuldade de movimento, quando comparados à faixa etária de 9 a 10 anos de idade. De acordo com Gallahue, Ozmun e Goodway (2013), na terceira infância, as crianças tornam-se mais fortes e rápidas, portanto as crianças da faixa etária entre 9 e 10 anos tendem a ser mais coordenadas do que as de 7 e 8 anos de idade. No Canadá, Rivard et al. (2014) encontraram menos dificuldades de movimento no desempenho motor de crianças mais velhas. Esses resultados explicam-se pelo aumento da proficiência motora conforme o aumento da idade (DAVIES; ROSE, 2000; DEBRABANT et al., 2012).

A detecção precoce do TDC é importante para a prevenção de possíveis impactos no dia a dia da criança, como nas atividades de autocuidado, desempenho acadêmico e atividades motoras (RIVILIS et al., 2011; AMERICAN..., 2013; BIEBARA et al., 2016), além das condiçôes de saúde, como no aumento do risco de depressão, ansiedade, obesidade, diminuição da autoestima e da aptidão física (CAIRNEY et al., 2010; ENGEL-YEGER; KASIS, 2010). Apesar de o presente estudo apresentar limitaçóes, tais como não ter feito medidas diretas aliadas ao MABC-2 e não ter incluído na amostra crianças de outras regióes do Brasil, destaca-se a importância dos resultados para contribuir na elaboração de estratégias para potencializar e estimular as capacidades motoras de crianças com desempenho motor abaixo do esperado.

Além disso, este estudo reforça a possibilidade da utilizaçáo de um teste motor que apresenta resultados seguros para a indicação de risco e dificuldade significativa de movimento considerando as características individuais de cada criança.

\section{Conclusão}

A prevalência do TDC, bem como as diferenças entre meninos e meninas, foi considerada semelhante aos achados das pesquisas internacionais. Os resultados também contribuem com os estudos nacionais, tendo em vista a existência de poucas pesquisas investigando o desempenho motor em amostras representativas. Estudos com delineamento longitudinal e intervençôes devem ser realizados em maior número. Além disso, sugere-se que futuros estudos controlem variáveis como, fatores de risco perinatais e condição socioeconômica.

\section{Referências}

AMERICAN PSYCHIATRIC ASSOCIATION - APA. Diagnostic and statistical manual of mental disorders. Arlington: American Psychiatric Publishing, 2013.

BIEBARA, E. et al. Manual function outcome measures in children with developmental coordination disorder (DCD): systematic review. Research in Developmental Disabilities, New York, v. 55, p. 114-131, 2016. 
BLANK, R. et al. European Academy for Childhood Disability (EACD): recommendations on the definition, diagnosis and intervention of developmental coordination disorder (long version). Developmental Medicine o Child Neurology, Malden, v. 54, n. 1, p. 54-93, 2012.

CAÇOLA, P. et al. Children with developmental coordination disorder demonstrate a spatial mismatch when estimating coincident-timing ability with tools. Research in Developmental Disabilities, New York, v. 48, p. 124131, 2015

CAIRNEY, J. et al. Developmental coordination disorder, self-efficacy toward physical activity, and play: does gender matter? Adapted Physical Activity Quarterly, Champaign, v. 22, n. 1, p. 67-82, 2005.

CAIRNEY, J. et al. Trajectories of relative weight and waist circumference among children with and without developmental coordination disorder. Canadian Medical Association Journal, Ottawa, v. 182, n. 11, p. 1167-1172, 2010.

CAPISTRANO, R. et al. Transtorno do desenvolvimento da coordenação e nível de atividade física em crianças: revisão sistemática da literatura. Cadernos de Terapia Ocupacional da UFSCar, São Carlos, v. 23, n. 3, p. 633646, 2015.

CHANG, S. H.; YU, N. Y. Comparison of motor praxis and performance in children with varying levels of developmental coordination disorder. Human Movement Science, New York, v. 18, n. 48, p. 7-14, 2016.

COUTINHO, M. T. C.; SPESSATO, B. C.; VALENTINI, N. C. Transtorno do desenvolvimento da coordenação: prevalência e dificuldades motoras de escolares da cidade de Porto Alegre. In: CONGRESSO BRASILEIRO DE CIÊNCIAS DO ESPORTE, 17., 2011, Porto Alegre. Anais... Porto Alegre: Colégio Brasileiro de Ciência do Esporte, 2011. Disponível em: <http:// congressos.cbce.org.br/index.php/conbrace2011/2011/ paper/view/3292>. Acesso em: 10 nov. 2015.

DAVIES, P. L.; ROSE, J. D. Motor skills of typically developing adolescents: awkwardness or improvement? Physical \& Occupational Therapy in Pediatrics, London, v. 20, n. 1, p. 19-42, 2000.

DEBRABANT, J. et al. Age-related differences in predictive response timing in children: Evidence from regularly relative to irregularly paced reaction time performance. Human Movement Science, Amsterdam, v. 31, n. 4, p. 801-810, 2012.

ENGEL-YEGER, B.; KASIS, A. H. The relationship between Developmental Co-ordination Disorders, child's perceived self-efficacy and preference to participate in daily activities. Child: Care, Health and Development, Oxford, v. 36, n. 5, p. 670-677, 2010.

FARHAT, F. et al. Assessment of physical fitness and exercise tolerance in children with developmental coordination disorder. Research in Developmental Disabilities, New York, v. 45, p. 210-219, 2015.
FARIAS JÚNIOR, J. C. et al. Physical activity practice and associated factors in adolescents in Northeastern Brazil. Revista de Saúde Pública, São Paulo, v. 46, n. 3, p. 505-515, 2012.

FIELD, A. Descobrindo a estatística usando o SPSS-2. Porto Alegre: Artmed, 2009.

GALLAHUE, D. L.; OZMUN, J. C.; GOODWAY, D. J. Compreendendo o desenvolvimento motor: bebês, crianças, adolescentes e adultos. São Paulo: Artmed, 2013.

GREEN, D. et al. The risk of reduced physical activity in children with probable Developmental Coordination Disorder: a prospective longitudinal study. Research Developmental Disabilities, Oxford, v. 32, p. 1332-1342, 2011.

HENDERSON, S. E.; SUGDEN, D. A.; BARNETT, A. L. Movement assessment battery for children. Londres: Pearson Assessment, 2007.

INSTITUTO BRASILEIRO DE GEOGRAFIA E ESTATÍSTICA - IBGE. Ensino: matrículas, docentes e rede escolar. Rio de Janeiro: IBGE, 2012. Disponível em: <http://www.cidades.ibge.gov.br/xtras/temas.ph p?lang $=\& \operatorname{cod}$ mun $=420540 \&$ idtema $=117 \&$ seach $=$ san ta-catarina|florianopolis|ensino-matriculas-docentes-e-rede-escolar-2012>. Acesso em: 5 nov. 2015.

JONGMANS, M.; SMITS-ENGELSMAN, B. C. S.; SHOEMAKER, M. M. Consequences of comorbidity of developmental coordination disorders and learning disabilities for severity and pattern of perceptual: motor dysfunction. Journal of Learning Disabilities, Austin, v. 36, n. 6, p. 528-537, 2003.

KITA, Y. et al. Applicability of the movement assessment battery for children-second edition to japanese children: A study of the age band 2. Brain and Development, Tokyo, v. 38, n. 8, p. 1-8, 2016.

LINGAM, R. et al. The association between developmental coordination disorder and other developmental traits. Pediatrics, Elk Grove Village, v. 126, n. 5, p. 1109 1118,2010

LUIZ, R. R.; MAGNANINI, M. M. F. A lógica da determinação do tamanho da amostra em investigaçóes epidemiológicas. Cadernos de Saúde Coletiva, Rio de Janeiro, v. 8, n. 2, p. 9-28, 2000.

MIRANDA, T. B.; BELTRAME, T. S.; CARDOSO, F. L. Desempenho motor e estado nutricional de escolares com e sem transtorno do desenvolvimento da coordenação. Revista Brasileira de Cineantropometria e Desempenho Humano, Florianópolis, v. 13, n. 1, p. 59-66, 2011.

NIEMEIJER, A. S.; WAELVELDE, H.; SMITS-ENGELSMAN, B. C. Crossing the North Sea seems to make DCD disappear: cross-validation of movement assessment battery for children-2 norms. Human Moviment Science, Amsterdam, v. 39, p. 177-188, 2015.

ORGANIZAÇÃO MUNDIAL DE SAÚDE - OMS. Classificação dos transtornos mentais e de comportamento 
da CID-10: descriçóes clínicas e diretrizes diagnósticas. Porto Alegre: Artmed, 1993.

ORGANIZAÇÃO MUNDIAL DE SAÚDE - OMS. Índice de Desenvolvimento Humano. Brasília, 2010. Disponível em: <www.pnud.org.br>. Acesso em: 13 maio 2016.

RIVARD, L. et al. Descriptive and factor analysis of the Developmental Coordination Disorder Questionnaire (DCDQ'07) in a population-based sample of children with and without Developmental Coordination Disorder. Child: Care, Health and Development, Malden, v. 40, n. 1, p. 42-9, 2014.

RIVILIS, I. et al. Physical activity and fitness in children with developmental coordination disorder: a systematic review. Research in Developmental Disabilities, London, v. 32, n. 3, p. 894-910, 2011.

SANTOS, V. A. P.; VIEIRA, J. L. L. Prevalência de desordem coordenativa desenvolvimental em crianças com 7 a 10 anos de idade. Revista Brasileira de Cineantropometria e Desempenho Humano, Florianópolis, v. 15, n. 2, p. 233-242, 2013.
SILVA, J.; BELTRAME, T. S. Indicativo de transtorno do desenvolvimento da coordenação de escolares com idade entre 7 e 10 anos. Revista Brasileira de Ciências do Esporte, Porto Alegre, v. 35, n. 1, p. 3-14, 2013.

SILVA, K. S. et al. Gender differences in the clustering patterns of risk behaviours associated with non-communicable diseases in Brazilian adolescents. Preventive Medicine, New York, v. 65, p. 77-81, 2014.

SOUZA, C. et al. O teste ABC do Movimento em crianças de ambientes diferentes. Revista Portuguesa de Ciências do Desporto, Porto, v. 7, n. 1, p. 36-47, 2007.

VALENTINI, N. C. et al. Prevalência de déficits motores e desordem coordenativa desenvolvimental em crianças da região Sul do Brasil. Revista Paulista de Pediatria, Sáo Paulo, v. 30, n. 3, p. 377-384, 2012.

VALENTINI, N. C.; RAMALHO, M. H.; OLIVEIRA, M. A. Movement assessment battery for children-2: translation, reliability, and validity for Brazilian children. Research in Developmental Disabilities, London, v. 35, p. 733-740, 2014

\section{Contribuição dos Autores}

Thais e Tailine foram responsáveis pela concepção, redação e revisão do texto. Renata, Érico e Rubian Diego foram responsáveis pela concepção, redação e revisão do texto e organização das análises. Juliano foi responsável pela concepção, redação e revisão do texto e formatação. Todos os autores aprovaram a versão final do texto.

\section{Fonte de Financiamento}

Coordenação de Aperfeiçoamento de Pessoal de Nível Superior (CAPES).

\section{Notas}

${ }^{1}$ Parte de bancos de dados de dissertaçôes de Mestrado apresentadas ao Programa de Pós-graduação em Ciências do Movimento Humano da Universidade do Estado de Santa Catarina. 